\title{
Defining Arrow of Time at the start of Inflation using Tradi- tional Cosmology Initially as an Example and Finally Penrose Cyclic Conformal Cosmology with Singular versus Nonsingu- lar Starting Points?
}

Andrew Beckwith ${ }^{1}$

1. Physics Department, Chongqing University, College of Physics, Chongqing University Huxi Campus, No. 44 Daxuechen Nanlu, Shapinba District, Chongqing 401331, People's Republic of China, rwill9955b@gmai.com, abeckwith@uh.edu

\begin{abstract}
:
We first of all define the arrow of time. Definition of the arrow of time will allow choosing different initial starting points. One of the issues we will also discuss is the interconnection between the arrow of time, entropy and quantum information. Seth Lloyd in his 2001 work made a linkage between entropy, bits, and information via an axiomatic approach involving time intervals. Our take is a bit more general. We will discuss as well the $t^{\prime} H o o f t$ 's statement as to initial conditions and times arrow, and how different cosmological models may influence initial conditions. Spoilers alert, if a nonsingular start to expansion existed, this would provide the most straightforward way to avoid a datum from classical relativity. That is, that in the actual equations of classical GR, there is no reason to have time asymmetry. Time asymmetry is built into initial conditions and we will detail several candidates. The first half of the paper brings up cosmology models and forming the arrow of time. The second is related to entropy itself and the problem of information.
\end{abstract}

Keywords: arrow of time; cosmological bounce; information; entropy; ccc cosmology (Penrose)

1. Introduction. Concerning the arrow of time and initial conditions in cosmology

In Cosmology, there is one outstanding datum, which is that in classical GR, outside of the initial conditions of the beginning of space-time, there is in reality no reason for times arrow. We will introduce times arrow, in the context of cosmology via initial conditions. But this means looking at 5 different sets of initial conditions. There are several which will be brought up. First the usual classical GR picture with a Penrose style singularity. Secondly, a nearly classical GR picture with a nonzero initial starting position[.The third is to use the Penrose CCC model while assuming a Penrose singularity theorem starting point. The fourth is to use the Penrose CCC model with a nonsingular start to the universe. The fifth is to look at a non singular start to the universe, with the ccc model generalized into a multiverse. These five cases will be brought up in terms of the initial conditions of the arrow of time, which we maintain should be in fidelity to the $t^{\prime}$ Hooft article's caution as to initial conditions. After stating the arrow of time, we will then bring up the issue of causal structure, and then causal relationships. This second big topic is one of information. The Lloyd mechanical engineering treatment of a strict bits of information connected with time has been around since 2001 and is criticized by cosmologists as to not taking into account the inflaton. A holographic universe description of information can also be utilized. One of our concluding remarks is in stating how 
our choice of inter relationship between entropy and information may affect initial conditions for the cosmological arrow of time.

\section{1s. . First defining a generic arrow of time}

Quote, from Eddington [ 1 ][2]

Let us draw an arrow arbitrarily. If as we follow the arrow we find more and more of the random element in the state of the world, then the arrow is pointing towards the future; if the random element decreases the arrow points towards the past. That is the only distinction known to physics. This follows at once if our fundamental contention is admitted that the introduction of randomness is the only thing which cannot be undone. I shall use the phrase 'time's arrow' to express this one-way property of time which has no analogue in space.

End of quote

The main point of this description is that time's arrow allegedly has only one way propertty of time as its conceptual starting point. Note in its formulation, the idea is of increasing randomness, which can be associated with entropy [ 3 ] In fact, [3] has given us an updated synopsis which we can bring up in the following

quote

Providing a microscopic expression for the entropy production has been one of the grand aims of statistical mechanics, going back to the seminal work of Boltzmann. However, both the range of validity of the second law and of its proposed derivations have, from the very beginning, generated discussion and controversy. The recent discovery of the fluctuation and work theorems has re-invigorated the debate

End of quote

In a word we have that the entire discussion of entropy, its production and all that start with the $2^{\text {nd }}$ law of thermodynamics [4], which we can simply state as

$$
\frac{d S(\text { entropy })}{d t} \geq 0
$$

Whereas the question raised, in [4] can be rendered in the following quote

This law is certainly not symmetric in time; if we interchanged past and future the entropy would tend to decrease. How did we get, from basic reversible equations, to a manifestly irreversible result?

End of quote

In [4] the author appeals to the existence of irreversibility due to initial conditions and this is his way out of this alleged conodrum as well does $t^{\prime}$ Hooft in a re work of the basic ideas as we can see in the following. [5]

Quote'

In the time-like direction, however, there cannot be complete symmetry. The universe appears to have started extremely small, conceivably it all started in a single point. That point must have been highly ordered, having total entropy very small or possibly zero. This is a reasonable boundary condition at the origin of time. Yet at the other end, when time grows to be very large, we see no need of any boundary condition; the universe may simply continue to expand forever, undergoing perpetual increase of entropy. Thus we have equations that are symmetric under time reversal but asymmetric in their boundary conditions. This suffices to explain the time asymmetry we see today.

\section{End of quote}

As it is, it depends upon initial conditions. In other words, if we take the words of [5] seriously, after the initial conditions, we have choices as to the formulation of the arrow of time. 
As a given we may consider what it takes to form initial conditions. One thought to keep in mind is that we will be, when establishing an order of time be affected, as brought up by $t^{\prime}$ Hooft[5]

Quote

As long as we adhere to the quantum mechanical description of all microscopical dynamical laws, we find the CPT theorem on our way, which implies that if we combine time reversal $T$ with parity reversal $P$ and particle-antiparticle interchange $C$, then this symmetry is perfect. We could well stick to our verdict that Nature's boundary conditions in the time direction suffice to explain the arrow of time

End of quote

In a word, we get times ARROW of time, going back to the ideas of Eddington[1], and [2] as a consequence of how we choose the initial conditions.

Perforce, we can choose our construction of initial conditions in the following set of examples, which correspond to different physical models for the cosmologies. Before we do so, there are several questions to ask

A. Is the beginning of space-time quantum mechanical at say the Planckian scale regime? Note Planck scale[6] refers to quantities of space, time, energy and other units that are similar in magnitude to corresponding Planck units' [7].

B. Where does the onset of causal structure [8][9][10]commence ?

C. Do we use as an example a thermal description of the generation of entropy in early universe cosmology [11][12] [13]?

D. Could Ng infinite statistics play a role in the counting of entropy in the early universe[14]

E. The Seth Lloyd treatment of Entropy, information, and how it may pertain to the arrow of time[15][16]

F. The Zeth treatment of time flow, [17]. This is appropriate if Time flow is directly correlated to entropy in the immediate onset of the universe. I.e. would the creation of the first time step in our universe correlate to the start of the Arrow of Time?

G. Other treatments as of Holographic generation of entropy and the idea of information [18]. This last idea is relevant if Gravitons, are a particle-wave entity and their mass consistent with a Ng Infinite Quantum statistic generation of a count of gravitons to create entropy generation. Highly speculative and saved for last

\section{Methods, Here we will be examining the different cosmological models and their relations to the 7 items above}

As stated in the beginning the first model to consider will be the Classical GR model, at the onset of inflation. We then look at different models in terms of the issues brought up in terms of if we have a singular start to the Universe, a nonsingular start to the universe, the Cyclic Conformal cosmology model in its Penrose version with a singular start to the Universe, CCC cosmology with a nonsingular start to the universe and then finally a multiverse model of the CCC cosmology with first an initial cosmology, and one with a nonsingular start to the Universe with a multiverse version of CCC cosmology, developed by the author

2.1. First let us consider the traditional model of the big bang and inflation. Does this have an arrow of time and generation of entropy as well as Causal structure and relations? 
At the moment of the Big Bang, almost all of the entropy was due to radiation, and the total entropy of the Universe was about $S=10^{88} \mathrm{k}$. Or slightly higher

There was a sea of particles, including matter, antimatter, gluons, neutrinos and photons, all around at energies billions of times higher than what the LHC can obtain today. There were so many of them -- perhaps $10^{\wedge} 90$ in total. If there was a traditional model of the big bang and inflation [19]

$S \sim 3 \frac{m_{\text {Plank }}^{2}\left[H=1.66 \cdot \sqrt{\tilde{g}_{*}} \cdot T^{2} / m_{\text {planck }} \cdot\right]^{2}}{T} \sim 3 \cdot\left[1.66 \cdot \sqrt{\tilde{g}_{*}}\right]^{2} T^{3}$

If we have a beach ball sized "universe" at the end of the inflationary era, with say tem-

perature of

$\mathrm{T}$ proportional to Planck temperature, of $\mathrm{T} 1.416785(71) \times 10^{32}$ kelvin we can approach $\mathrm{S}=$

$10^{88} \mathrm{k}$ B On the other hand, we may have a value slightly larger. Is this due to thermal versus

particle generation?. If there was a traditional model of the big bang and inflation [19] We will then have the situation which has Eq.(2) holding due to superhot Plankian

temperatures holding where we also would have $g_{*}$ being the initial degrees of freedom which according to Kolb and Turner [20] would take the value of about 100 to 120, whereas Beckwith postulated in [20] a value up to 1000. The interested reader can ascertain this value. What we should not ignore is that this value of Eq.(2) of about $10^{\wedge} 88$ to $10^{\wedge} 90$ is commensurate, if we look at the $\mathrm{Ng}$ counting algorithm of This discussion above is to re-

mark

on the importance of an initial process for the onset and the growth of entropy .

To measure entropy in cosmology we can count photons. If the number of photons in a

given

Volume is $\mathrm{N}$, then the entropy of that volume is $\mathrm{S} \sim \mathrm{kN}$ where $\mathrm{k}$ is Boltzmann's constant

Note that Y. Jack Ng. has [14], from a very different stand point derived $S \sim n$ based upon string theory derived ideas, with $\mathbf{n}$ a 'particle' count, which in Y. Jack Ng's procedure is based upon the number of dark matter candidates in a given region of phase space..Y. Jack $\mathrm{Ng}^{\prime}$ s idea was partly based upon the idea of quantum ' infinite ' statistics, and a partition function

In the Traditional inflationary model, we have at some time during the inflationary period that if the temperature reaches Plank value which may allow for a match up of entropy according to $\mathrm{Eq}(2)$ of about $10^{\wedge} 88$ to $10^{\wedge} 90$ due to either Plank temperature and / or primordial "particle" count of $10^{\wedge} 88$ to $10^{\wedge} 90$

The problem arises though, if one has a SINGULARITY as to how and where the arrow of time will be initiated. In [21] Andreas Albrecht tries to reconcile the traditional picture of entropy generation and the arrow of time as stated in is abstract

Quote 
Cosmic inflation claims to make the initial conditions of the standard big bang "generic". But Boltzmann taught us that the thermodynamic arrow of time arises from very non-generic ("low entropy") initial conditions. I discuss how to reconcile these perspectives. The resulting insights give an interesting way to understand inflation and also compare inflation with other ideas that claim to offer alternative theories of initial conditions.

End of quote

His key statement as to the Arrow of time is the following'

Quote

The arrow of time, as it is currently understood, simply has to be used as an "input" to any theory of the universe. At its most fundamental level, the arrow of time emerges from evolution from a special initial state toward more generic subsequent states (where "generic" and "non-generic" are defined relative to the natural evolution under the equations of motion and also relative to a particular coarse-graining). To have an arrow of time, there must be something non-generic about the initial state. That property of the initial state must be chosen, not because it is a typical property but because that (necessarily atypical) property is required in order to have an arrow of time

End of quote

We submit that as clever as [21] is as far as postulating the input parameter that there is not a clear path as to creating an initial state, as the onset of inflation satisfying the Boltzman formulation of an arrow of time. We could avoid this difficulty if we may start from either a cold, to a hot temperature, thereby satisfying Eq (2), or if there is in the vicinity of Eq.(2) a linkage to a counting algorithm.; I.e. Boltzman entropy and the arrow of time would imply the existence of a colder than Planck temperature leading to its subsequent rise later on.

In other wise, in the traditional model of inflation gives a muddled initial rise to the initial conditions which would permit an arrow of time to arise in the first place. Reference [21] actually highlights the difficulty which is in specifying non generic initial conditions. Whereas these are not

\subsection{Considering an arrow of time arising from a quantum bounce or its equivalent condition and a nonsingular inflationary start}

This idea of a nonsingular start is exemplified by Freeze et.al. as in

$$
\left(\frac{\dot{a}}{a}\right)^{2}=H_{\text {bounce-cos }}^{2}=\frac{8 \pi}{3 M_{P}^{2}} \cdot\left(\rho-\frac{\rho^{2}}{2|\sigma|}\right)
$$

(3)

Equation (3) has the density, as given by $\rho$ altered by the existence of a wall for the nonsingular start of expansion which has a tension value of $\sigma$ i.e. Eq. (3) is zero when the density $\rho$ is equal to 2 times the absolute value of $\sigma$, and Freeze in [22] in page 153 of the book [22] refers to $\sigma$ as proportional to at least Planck mass $M_{\text {planck }}$, or of the value of $\mathrm{TeV}$ 
Left unsaid, in this is a causal mechanism for the introduction of a low entropy to a rapidly climbing value of entropy, so as to initiate the arrow of time, in line with the discussion of that problem given in [21] which we quoted earlier.

In [22], there is a reference to the destruction of primordial black holes which is given as when the density of universe climbs to a value given as $\omega_{Q}=p_{Q} / \rho_{Q}$ is defined, with the numerator being the pressure, and denominator density of phantom fields . which leads to by [22] a density for which there is breakup of primordial black holes

$\rho_{B H} \approx M_{p}^{4} \cdot\left(\frac{M_{p}^{2}}{M^{2}}\right) \cdot\left(\frac{3}{32 \pi}\right) \cdot \frac{1}{\left|1+\omega_{Q}\right|}$

If the black holes being broken up lead to particle generation, which could then feed into writing say

$$
S_{\text {bounce }} \approx n_{Q}=\text { Gravitons }- \text { from }- \text { black }- \text { holes }
$$

The problem would then be to delineate conditions for which the Eq.(5) would lead from a low to a high entropy build up, which would require a lot of computer simulation work to ascertain, but it may, if done carefully yield conditions as to the causal conditions for creation of an arrow of time;. The problem would be then to ascertain if and when the causal conditions lead to the density of the Universe yielding a value say of the order of magnitude of Eq.(4) above

Keep in mind that according to [ 23 ] Khlopov, has the following for black hole density, namely

$\rho_{B H} \approx \frac{M}{\left(r_{g}=2 G M / c^{2}\right)^{3}} \equiv \frac{c^{6}}{8 G^{3} M^{2}}$

Here, $M$ is the presumed mass of a black hole, and the result is counter intuitive to say the least, as $r_{g}$ is the mass of the configuration with mass $\mathrm{M}$

We state that in this situation we have that there may be

$$
S_{\text {gravitons }} \approx n_{\text {gravitons }} \propto S_{\text {Thermal }} \approx T_{\text {thermal-temp }}^{3}
$$

But this depends upon having

$$
\rho_{B H} \approx \frac{c^{6}}{8 G^{3} M^{2}} \approx \frac{3 M_{P}^{6}}{32 \pi M^{2}\left|1+\omega_{Q}\right|}
$$

If we use $\left|1+\omega_{Q}\right| \approx \frac{3}{4 \pi}$ and $M_{P}=G=c=1$, we have a $\omega_{Q} \approx-\left(\frac{4 \pi-3}{4 \pi}\right)$ so that then pressure and density are approximate negative values of each other, which is implying the following. i.e. The cosmological constant has negative pressure, but positive energy. The negative pressure ensures that as the volume expands then matter loses energy (photons get red shifted, particles slow down); this loss of energy by matter causes the expansion to slow down - but the increase in energy of the increased volume is more important. The increase of energy associated 
with the extra space the cosmological constant fills has to be balanced by a decrease in the gravitational energy of the expansion - and this expansion energy is negative, allowing the universe to carry on expanding. If so then we can say that having Eq. (7) means we are having a cosmological constant argument

The existence of the cosmological constant, i.e. DE, does not mean though that we have satisfied the conditions for the existence of the arrow of time. I.e. even if Equation (7) holds it is not clear from our argument exactly when we would have that

$S_{\text {gravitons }} \approx n_{\text {gravitons }} \propto S_{\text {Thermal }}$

In order to have the value of the increasing onset of the entropy we would like to have the following, namely'using Eq.(1) we would assert a causal ordering following the given values of:

$S+\Delta S \approx n+\Delta n \geq n$ iff $t+\Delta t \geq t$

This above would be in itself proof of the existence of an arrow of time, being initiated in early universe conditions

To get Eq.(10) in the case of a single universe would require having an increasing entropy with low entropy at the boundary of the causal bubbler, for a particular solution to Eq. (1) above. This may or may not be allowed if we have, with a quantum bounce entropy where $\mathrm{Eq}(9)$ and Eq.(10) are consistent if and only if we have an increase in temperature generating entropy.

What allows such a construction even if we have a quantum bounce? As given by Hawking, in [24]

\section{Quote}

The observed asymmetry or arrow of time defined by the direction of time in which entropy increases is shown to be related to the cosmological arrow of time defined by the direction of time in which the Universe is expanding. It arises because in the proposed quantum state the Universe would have been smooth and homogeneous when it was small but irregular and inhomogeneous when it was large $\backslash \backslash$

\section{End of quote}

The additional proviso is that there be an expansion of the Universe, as given by the 1985 paper by Hawking, in [24] whereas in [25]

Quote

We have also pointed out that the emphasis on the second law of thermodynamics owes its origin to the assumption that only a time-reversal non-invariant law can provide the appropriate starting point for solving the problem of the arrow of time. The lawlike and the de facto approaches share this assumption; the difference between them lies in the fact that the latter one attempts to reduce the second law to the fundamental laws of mechanics -classical on quantum- plus some restriction on initial conditions

End of quote 
In [25] the aftermath of this citation is to refer to ontological arguments as to remove this adherence to the above paragraph

This is flatly contradicted by 'tHooft, in [5] and given the relative import of the author of [5] versus the citation of [25] I as an author would tend to agree with 'tHooft and not [25].

We can though as a wrap up state that even if we have a cosmic bounce, that

A. Time ordering of say time $t A$, to time $\mathrm{tB}$ being congruent with increasing time which is commensurate with an expansion of the universe

B. The increase in time, from time tA to time tB would be commensurate with an increase in entropy

C. Initial conditions specifying A and B which would then get around what cosmologist Krasnikov [26] brought up pertinent to the nonapplicable nature of the arrow of time argument in classical general relativity equations outside the initial conditions which will be used to specify by use of casual relations time ordering conditions which would introduce the arrow of time.

D. If A and B are satisfied, it would also be relevant to have a thermal condition of increasing temperature from time $t A$ to time $t B$ which would be consistent with an increase of entropy

E. For those who wish to have strict fidelity with regards to Boltzmans formulation of entropy, independent of the arrow of time issue, the author refers readers to [27], pages 521-561

F. For the record, the author states that we do not have a replacement for Boltzman's formulation of entropy, as given in Eq. (1) provided that our cosmology models are closed, thermodynamically. In terms of what [26] brought up as well as $t^{\prime} \mathrm{Hooft}$ in [5] , the nongeneral, special solution approach should be used. This in itself would be enough to start the arrow of time.

\subsection{Considering now what happens if we have $M_{P}=G=c=1$, and look at the classical Penrose Cyclic Conformal Cosmology model, with a singular starting point for the present universe}

Penrose CCC theory, is in its most basic formulation given in the following

The main methodology in the Penrose proposal has been in Eq. (11) evalu-

ating a change

$$
\begin{aligned}
& \text { in the metric } g_{a b} \text { by a conformal mapping } \hat{\Omega} \text { to [28] } \\
& \hat{g}_{a b}=\hat{\Omega}^{2} g_{a b}
\end{aligned}
$$




$$
\hat{\Omega} \underset{c c c}{\longrightarrow} \hat{\Omega}^{-1}
$$

The sum effects of Eq. (11) and Eq. (12) are to have matter-energy swept up by supermassive black holes, and to have this block of matter-energy recycled back into a new starting point for a big bang. This is an alternative to the idea of other oscillating universe models[29] [30]which commonly had the problem brought up by Tolman in the 1930s, in which oscillating models would per cycle have a steadily increasing entropy budget at the start of expansion of the universe per universe cycle[29][30]. The author has generalized this for reasons which will be stated in the future part of this reference.

Good news about the Penrose hypothesis, it removes the Tolman increasing entropy fiasco [29][30]. The bad news is that it does nothing to ensure the consistency of parameters of physics per creation cycle

While the Penrose idea is truly bold and visionary[28], the singular start to the universe does nothing to alter the problems as to forming an arrow of time for a singular starting point. I.e. all the objections and cautions as of section 2.1 apply here, as there is also yet another hazard as there are in this model no restraints as to altering the fundamental constants of physics, i.e. we get then the problem that Planck's constant could change from one cycle to another. i.e. this unacceptable situation. As stated in [31], the following could be disastrous. It also leads to [32] and we did [33] as corrections to Eq.(13)

$\hbar_{\text {Prior-universe }} \neq \hbar_{\text {present-universe }}$

Needless to say, having Equation (13) true would definitely upend physical law. If one wants to see how unacceptable Eq. (13) is, look at [31] where the authors do a credible explanation as to how even small variations as indicated in Eq. (13) could make life impossible. Even worse are discussions as to Hawking and other authors of the idea of "baby universes" and Darwinian selection of a range of values for baby universes where only a few would survive. The long and short of it is that [29][30][31] is a prescription for a nonstable cosmological order, which would eventually exhaust itself and not be eternal, by a long shot[32]

\subsection{What if there is Penrose CCC cosmology? With a nonsingular beginning ? How would that affect the arrow of time, if there is a singular start to the universe we inhabit?}

This idea is very similar to Section 2.2, with the proviso of no build up of entropy, if there is a repeated universe, but then we have the added likelihood of having a satisfaction of the 'tHooft rules of [5] , i.e. we have special solutions for introducing time arrow asymmetry

In addition though we still could have Equation (13) with all its attendant fiascos as given in [31]. Since this would be a repeating SINGLE universe, some version of Eq.(1) would hold, even if we had the problem of Eq. (13) reappearing[31][32]

The change of satisfying [5] strictures as to the start of an arrow of time would actually increase over what was stated in section 2.3 , but we still could have the Eq. (13) fiasco 
2.4 What if there is a multiverse generalization of Penrose CCC cosmology, with a singular start to the universe?

We will be then going to [33] and its multiverse generalization. That would solve the problem of Eq. (13) and insure the continuation of physical law from cycle to cycle, but we would have perhaps difficulties in satisfying [5]. The following discussion from [33] would keep the consistency of physical law from cycle to cycle and avoid the Tolman fiasco of increasing entropy[29] [30]per cycle of creation

In this situation we will be assuming the mechanics of Eq. (12) and Eq. (13)

with a

twist added. We have not one universe, but a plethora of universes, of some

num

verse

number, $N$, which would be via averaged out partition functions of the uni-

contributing to a new universe cycle using Eq. (12) and Eq. (13) with [31][32]

avoided

The reader is referred to [33] and we will cite with attribution to [33] the following multiverse generalization while still maintaining the Penrose Singularity theorem[34][35]

We now outline the generalization for Penrose CCC(Cosmology) [33] just before inflation which we state we are extending Penrose's suggestion of cyclic universes,

black

hole evaporation, and the embedding structure our universe is contained within,

This multi

there

verse has BHs and may resolve what appears to be an impossible dichotomy .That

are $\mathrm{N}$ universes undergoing Penrose 'infinite expansion' [33] contained in a mega universe structure[33]. Furthermore, each of the $\mathrm{N}$ universes has black hole evapo-

ration, . If

each of the $\mathrm{N}$ universes is defined by a partition function[33][36], called $\left\{\Xi_{i}\right\}_{i=N}^{i=1}$,

then there

$10^{7}-10^{8}$

exist an information ensemble of mixed minimum information correlated about

bits of information per partition function [33][36] in the set $\left.\left\{\Xi_{i}\right\}_{i=N}^{i=1}\right|_{\text {before }}$, so mini-

mum in

formation is conserved between a set of partition functions per universe [33][36]

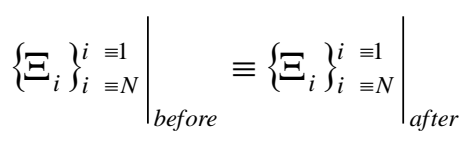

However, there is non-uniqueness of information put into partition function

$\left\{\Xi_{i}\right\}_{i=N}^{i \equiv 1}$.Also 
Hawking radiation from black holes [33]is collated via a strange attractor collection

in the mega

universe structure to form a new inflationary regime for each of the $\mathrm{N}$ universes

represented

Note that in [ 37 ] that there is an upper bound as to the number of universes in a

multiverse

In addition we have that each partition function [33][36]

$$
\left\{\Xi_{i}\right\}_{i \equiv 1}^{i \equiv N} \propto\left\{\int_{0}^{\infty} d E_{i} \cdot n\left(E_{i}\right) \cdot e^{-E_{i}}\right\}_{i \equiv 1}^{i \equiv N}
$$

Each of $E_{i}$ identified with Eq.(15) above, are with the iteration for $\mathrm{N}$ universes [34],

Then the following holds, by asserting the following claim to the universe as a mixed

state

with black holes playing a major part, i.e. we are doing an averaging procedure to

remove the

Anthropic principle[33]via

\section{CLAIM 1}

See the below[33] representation of mixing for assorted $\mathrm{N}$ partition function per CCC

cycle

$$
\left.\left.\frac{1}{N} \cdot \sum_{j=1}^{N} \Xi_{j}\right|_{j \text {-before-nucleation-regime }} \stackrel{ }{\text { vacuum-nucleation-tranfer }} \longrightarrow \Xi_{i}\right|_{i \text {-fixed-after-nucleation-regime }}
$$

Furthermore, the main point is done in [33] in terms of general ergodic mix-

$\operatorname{ing}[38][39][40]$

[41] is given by using the Penrose ccc idea in modified via ergodic mixing

2.5. CCC cosmology in multiverse form with nonsingular start to the universe, and the arrow of time 
We have the advantages of removing variation of cosmological parameters like Planck's constant so the following is maintained without [31] and [32] due to multiverse CCC cosmology. If so then,

$$
\hbar_{\text {Prior-universe }}=\hbar_{\text {present-universe }}
$$

Also as in 2.3 AND 2.4, we do not have a build up of entropy per cycle as in [26] due to the CCC cosmology being used directly

Now we need to have a way to create initial configuration steps to an arrow of time

To do this we will be examining a way to have the criteria of [5] satisfied via a multiverse solution to the cosmological constant problem and rely upon gravitons as the square root of the cosmological constant (assuming massive gravity) as to right after the assumed bubble solution as referenced in Eq. (3) above

Let us first solve the cosmological constant problem via the Penrose multiverse construction and link that to massive gravitons which will in this final iteration serve as the particular solution to a graviton count which will serve as our working hypothesis as to entropy and initial conditions which will then be the basis of our arrow of time

2.5.1 Creating a cosmological constant and its linkage to gravitons, for counting algorithm and the start of the arrow of time

We will first start off with the redone calculation as to the Vacuum energy as given in [42] and how we rescale them to be in sync as to the observed experimental value for vacuum energy which is of the present era. This methodology is consistent with the Zero-point energy calculation, we start off with the following as given by [42]

$$
\begin{aligned}
& \frac{1}{2} \cdot \sum_{i} \omega_{i} \equiv V(\text { volume }) \cdot \int_{0}^{\hat{\lambda}} \sqrt{k^{2}+m^{2}} \frac{k^{2} d k}{4 \pi^{2}} \approx \frac{\hat{\lambda}^{4}}{16 \pi^{2}} \\
& \underset{\hat{\lambda}=M_{\text {Planck }}}{\longrightarrow} \rho_{\text {boson }} \approx 2 \times 10^{71} \mathrm{GeV}^{4} \approx 10^{119} \cdot\left(\rho_{D E}=\frac{\Lambda}{8 \pi G}\right)
\end{aligned}
$$

In stating this we have to consider $\rho_{D E}=\frac{\Lambda}{8 \pi G} \approx \hbar \cdot \frac{(2 \pi)^{4}}{\lambda_{D E}{ }^{4}}$, so then we have to

consider a wavelength $\lambda_{D E} \approx 10^{30} \ell_{\text {Planck }}$ which is about $10^{30}$ times a Planck length radius of a space-time bubble [33] as a nonsingular expansion point for Cosmology, at the start of inflation with the space-time bubble of about a Plank length radius in size. . Having said that, how do we get having the Penrose result modified into a multiverse condition[33]

$$
\lambda_{D E} \approx 10^{30} \ell_{\text {Planck }}
$$

before the near singularity is to be solved. Then the existence of solves the problem.

$$
\rho_{D E}=\frac{\Lambda}{8 \pi G} \approx \hbar \cdot \frac{(2 \pi)^{4}}{\lambda_{D E}^{4}}
$$

This happens, if we use the value of Eq. (19) fully consistent with regards to a value in line 
with the DE density seen today, i.e. cutting the value of Eq. (18) by $10^{\wedge} 120$ or more . In this we have to consider the value of the mass of a graviton by [43] as seen by making use of the following cosmological formulas. First the cosmological scale factor as seen by [44], in equation (21), then an inflaton energy density given by equation(22), and the Mass of a graviton given in Eq. (23), and the Dark energy wavelength Eq.(24). All of these will be referred to in the remainder of this document while keeping in mind the big result from [43]

$$
\begin{gathered}
a(t)=a_{\text {initial }} t^{\gamma} \\
\rho \approx \frac{\dot{\phi}^{2}}{2}+V(\phi) \equiv \frac{\gamma}{8 \pi G} \cdot t^{2}+V_{0} \cdot\left\{\sqrt{\frac{8 \pi G V_{0}}{\gamma(3 \gamma-1)}} \cdot t\right\}^{\sqrt{\frac{\gamma}{4 \pi G}}-\sqrt{\frac{8 \pi G}{\gamma}}} \\
m_{g}=\frac{\hbar}{c} \cdot \sqrt{\Lambda} \\
\lambda_{D E} \approx 10^{30} \ell_{\text {Planck }}
\end{gathered}
$$

To make sense of the above, it is useful to keep in mind the following. If we have a cosmic start by initially calling the Pre - Planckian geometry as 4 space, with no (in our universe any reference point) time axis. Before the transferal to our present universe we have the following identification, namely 4 (space) + no time becomes 3+1 (3 space, one time) in the transfer from the prior universe construction to our own cosmological constant. I.e. before, in Pre Planckian space-time, time as we know it really does not exist

\subsubsection{Constructing a counting algorithm for setting up an arrow of time, using our} boundary conditions for a nonsingular multiverse

entropy

The idea is to use the idea of $\mathrm{Ng}[14]$ directly, and in doing so, to make the initial buildup of

monotonically increasing in conjunction with an increase in space-time volume. This would assume that past a causal boundary which we will specify the use of Eq.(1) so that there is' of space-time in which one would have entropy steadily increasing. There are two ways to do

this

mixing of

procedure. First of all we examine conditions leading to a particle count of entropy due to the the creation of conditions for a graviton mass, due to graviton mass being proportional to the square root of a derived cosmological constant. Way two is to go to Eq.(8) satisfied for the value of $\omega_{Q}$

which

will have pressure and density satisfied by emergent inflaton physics. In lieu of constant and unending crticism of Seth Lloyd [15] who does not use inflaton physics, the author commits himself to a future lemma to be shown in terms of researchwork, namely

Lemma1

The value of Eq.(22) becomes effectively having "final density" $\rho_{f}=\rho(E q .22)$ and 
we make the approximation $H_{\text {bounce-cos }}^{2}=\frac{8}{3 M_{P}^{2}} \cdot\left(\rho-\frac{\rho^{2}}{2|\sigma|}\right)=0 \Rightarrow \rho=2|\sigma| \geq 2 M_{P}$ i.e.

twice

Plank mass , or greater as also equal to if we have Plank length with a thermal energy like[44]

2 times Planck mass $=\rho \approx \frac{\dot{\phi}^{2}}{2}+V(\phi) \equiv \frac{\gamma}{8 \pi G} \cdot t^{2}+V_{0} \cdot\left\{\sqrt{\frac{8 \pi G V_{0}}{\gamma(3 \gamma-1)}} \cdot t\right\}^{\sqrt{\frac{\gamma}{4 \pi G}}-\sqrt{\frac{8 \pi G}{\gamma}}}$

Picking positive pressure and initially negative energy, as to what was given in Rosen[ 45 ]

We can compare this closed within a causal wall initial configuration start with the

Rosen[ 32] value of energy for a mini universe

(from a Schrodinger equation) with ground state mass of $m=\sqrt{\pi} M_{\text {Planck }}$ and energy of

$$
E_{\hat{n}}=\frac{-G m^{5}}{2 \pi^{2} \hbar^{2} \widehat{n}^{2}}
$$

Our preliminary supposition is that Eq. (26) could represent the initial energy of a Pre Planckian Universe and that thermal energy is dumped in due to the use of Cyclic Conformal cosmology ( maybe in multiverse form) so that if there is a build up of energy greater than Eq.(26) due to thermal buildup of temperature due to infall of matter-energy, we have a release of Gravitons in great number which would commence as a domain wall broke down about in the Planckian era with a temperature of the magnitude of Planck Energy for a volume of radius of the order of Plank Length. Now for using Eq. (26), we look at [46], where we have then an information number of N(information) for which we have a total Graviton mass of

$$
M_{\text {graviton-total }}=n_{\text {graviton }} \cdot m_{\text {graviton }}
$$

Where we will be looking at a value of "information" of initially[25]

$$
\mathbb{N}(\text { inf })=\frac{9 \pi}{\ln 2} \cdot\left(\frac{n_{\text {graviton }} \cdot m_{\text {graviton }}}{m_{\text {planck }}}\right)^{2} \approx \frac{9 \pi \cdot\left(n_{\text {graviton }}\right)^{2}}{\ln 2} \cdot 10^{-120}
$$

Now use the following approximation of the Universe, initially having the entropy of a black hole, i.e, we are using Ng Infinite Quantum statistics, [14]

$$
S_{\text {Universe }} \propto S_{B H} \cong \frac{A(\text { area })}{4 \cdot \ell_{\text {Planck }}^{2}} \approx \frac{9 n_{Q}}{4} \approx n_{\text {graviton }}
$$


In taking this step, we are making use of [45] having the following radius used, namely using in our model of a black hole, the quantum "atom" approximation

$$
r\left(n_{Q}\right) \approx \frac{3 \sqrt{n_{Q}} \cdot \ell_{\text {Planck }}}{2 \sqrt{\pi}}
$$

In order to have non vanishing information according to [45] [46] we would need to specify

$$
\begin{aligned}
& \mathbb{N}(\text { inf })=\frac{9 \pi}{\ln 2} \cdot\left(\frac{n_{\text {graviton }} \cdot m_{\text {graviton }}}{m_{\text {planck }}}\right)^{2} \approx \frac{729 \pi \cdot\left(n_{Q}\right)^{2}}{16 \cdot \ln 2} \cdot 10^{-120} \geq 1 \\
& \Rightarrow E_{\hat{n}=n_{Q}}=\frac{-G \cdot\left(m=\sqrt{\pi n_{Q}} \cdot m_{\text {planck }}\right)^{5}}{2 \pi^{2} \hbar^{2} n_{Q}^{2}} \approx \frac{-G \cdot\left(\sqrt{\pi n_{Q}} \cdot\left(m_{\text {planck }}\right)^{5}\right)}{2 \hbar^{2}} \\
& \underset{h=m_{\text {planck }}=G=1}{\longrightarrow} E_{\hat{n}=n_{Q}} \simeq \frac{-\left(\sqrt{\pi n_{Q}} \cdot\right)}{2} \approx l \arg e-\text { negative-value }
\end{aligned}
$$

The idea in all of this, is that this initial configuration would require a very high thermal value, with the thermal

value

such that when the thermal wall of the nonsingular start to expansion i.e. we would have a positive energy due to

thermal inputs from cyclic conformal cosmology so that the thermal value would be greater than $\mathrm{Eq}(31)$ negative

energy in order to have information inserted into the new universe

This leads to a lemma 2, to be proved in subsequent work

$\underline{\text { LEMMA } 2}$

We will set the minimum energy $\Delta E \quad$ as greater than or equal to the magnitude of the negative energy

given in Eq.(31) with a minimum time step defined as follows

$$
\Delta t \approx \frac{\hbar}{\Delta E}
$$

The term $\Delta E \approx|E n e r g y-i n-E q .(31)|$ with entropy defined via $E q(29)$, i.e. a 2 to one

Proportinality in terms of quantum numbrer, and count of gravitons. If the Gravitons increased In number, this would be an increase in entropy which would fufil the necessary conditions of Times arrow having a direction specified by increasing entropy 
3. COMPARING TIMES ARROW as being created by a threshold information release criteria as compared to Seth Lloyd's linkage of entropy and bits of information

Seth Lloyd in 1999 [15] obtained the following and this is to a certain degree duplicated in [19] and it has

limitations

A way to obtain traces of information exchange, from prior to present universe cycles is finding

lyzed, then

linkage between information and entropy. If such a parameterization can be found and ana-

Seth Lloyd's [15] shorthand for entropy,

$$
I=S_{\text {total }} / k_{B} \ln 2=[\# \text { operations }]^{3 / 4}=\left[\rho \cdot c^{5} \cdot t^{4} / \hbar\right]^{3 / 4}
$$

could be utilized as a way to represent information which can be transferred from a prior to

the present

carriers, and

universe . The question to ask, if does Eq. (33) permit a linkage of gravitons as information can there be a linkage of information, in terms of the appearance of gravitons in the time interval of, say

$0<t<t_{\text {Planck }}$ either by vacuum nucleation of gravitons / information packets

Oops. What is the problem ? There is no idea as to making a linkage between a pre-universe

set of

early

to [5]

Arrow of

initial conditions, and if one has enough linkage to likely inflaton physics to make analysis of universe conditions. Seth Lloyds Eq. (33) idea of a construction of entropy has no relationship where 'tHooft specified initial conditions, which are not general to give us the start of the time

\section{Discussion}

The way to extend this inquiry is to make a data set comparison of collection of bins of early universe GW 'information' and to ascertain if there is indeed the threshold effect alluded to in Lemma 1 and Lemma 2. Doing so would give us

Conformation as to this inquiry and give a range of energy values to consider for threshold effects alluded to in Lemma 1 and Lemma 2 for the Penrose Cyclic conformal cosmology in multiverse form given in this document. The author also wishes to make reference as to a completely different take on Multiverse physics 
usually taken up whereas there is an extremely unlikely value given as to the existence of the probability of a multiverse state having a given value of $\Lambda$ via Hartle-Hawking theory having a given probability of the square of the Hartle-Hawking wavefunction

i.e.

$$
P(\text { probability }) \sim \exp \left(-24 \pi^{2} / \Lambda\right)=\exp \left(-S_{A}\right)
$$

This probability would lead to a ridiculously large time value one would have to wait for any such occurrence happening in the multiverse[47]

In essence, the String theorists as well as Hartle and Hawking have convinced themselves as to the extreme unlikelihood of any identified state in the multiverse which we view as a misuse of the existence of the cosmological constant. Our work has avoided this stunning result which we view as not linking the existence of a cosmological constant with starting the arrow of time and entropy generation

This probability would lead to a ridiculously large time value one would have to wait for any such occurrence happening in the multiverse that a vacua would have a cosmological constant with our known value in our universe of $\Lambda$. This allows us to state that in our universe we live in a universe with the smallest non zero value of $\Lambda$ but makes its choice and eventual emergence in a universe almost non existent

In essence, the String theorists as well as Hartle and Hawking have convinced themselves as to the extreme unlikelihood of any identified state in the multiverse which we view as a misuse of the existence of the cosmological constant. Our work has avoided this stunning result which we view as not linking the existence of a cosmological constant with starting the arrow of time and entropy generation

\section{CONCLUSION}

The author found several interesting datum out which should be enumerated for the benefit of the reader

A. First of all, the classic one universe application of cyclic conformal cosmology appears to remove the Tolman catastrophe as to build up of entropy per cycle.[29]

B. Having a singular start to the universe, via a Penrose style singularity does not appear to satisfy the 'tHooft [5] requirement as to a particular, not a general solution to have initial conditions permitting a boundary value beginning to the Arrow of time. The fault appears to lie in that there is a thermal component to entropy generation which would entail a rise in initial temperature which does not seem to be the case. Hence we ruled this out. If there is no classical Penrose CCC recycling, there appears thermally no way as to have rising temperatures at the start of inflation

C. Having a multiverse CCC (generalization of Penrose CCC) appears to allow for satisfying having Eq.(17) hold

D. The Seth Lloyd treatment of entropy, and its relation to bits (computationally) appears to be in violation of [5], but we do have a way not in- 
volving Eq. (33) which is given by Eq. (32) which would also involve inflaton physics, plus the discussion of utilizing information via [45] and also [46]

E. Reference [47] and Eq. (34) appear to make the Hartle-Hawking wavefunction of the universe, when generalized leaving an infinitesimal probability of finding via the probability of having a vacua (defined as a universe in a multiverse ) with a cosmological constant $\Lambda$ via traditional string theory methods. This is akin to the 'baby universe' argument and the probability is so low that one would have to wait well over a trillion years for this to occur in the generation of a universe with a fixed $\Lambda$

F. Our method instead, with thermal averaging as given by [33] uses a stable $\Lambda$ value to calculate gravitons, with mass to generate entropy and likely the solution to [5] being matched up in a use of the procedure given in [18] and also [43]

G. With all of this, we commence our review of five different cosmologies and their impact on the arrow of time. Casual relations and casuality is established in the final fifth cosmological model, while assuming a nonsingular start to expansion of the universe to satisfy [5]

Conflicts of Interest: "The authors declare no conflict of interest."

\section{References:}

1. Weinert, Friedel (2005). The scientist as philosopher: philosophical consequences of great scientific discoveries. Springer. p. 143. ISBN 978-3-540-21374-1., Chapter 4, p. 143

2. Eddington, , Sir Authur, https://henry.pha.jhu.edu/Eddington.2008.pdf

3. I M R Parrondo, C Van den Broeck and R Kawai, "Entropy production and the arrow of time", New Journal of Physics, Volume 11, July 2009, J M R Parrondo et al 2009 New J. Phys. 11073008

4. Rudolph Pierels, "Surprises in Theoretical physics" Princeton Series in Physics, Princeton, New Jersey, USA, 1979

5. Gerard 't Hooft', "time, the Arrow of time and Quantum Mechanics", Front. Phys., 29 August 2018 I https://doi.org/10.3389/fphy.2018.00081

6. John D. Barrow, 2002. The Constants of Nature; From Alpha to Omega - The Numbers that Encode the Deepest Secrets of the Universe. Pantheon Books

7. Planck, Max (1899). "Über irreversible Strahlungsvorgänge". Sitzungsberichte der Königlich Preußischen Akademie der Wissenschaften zu Berlin (in German). 5: 440-480. pp. 478-80 contain the first appearance of the Planck base units other than the Planck charge, and of Planck's constant, which Planck denoted by $b . a$ and $f$ in this paper correspond to $\underline{k}$ and $\underline{G}$ in this entry

8. A.V. Levichev; Prescribing the conformal geometry of a Lorentz manifold by means of its causal structure; Soviet Math. Dokl. 35:452-455, (1987)

9. Alfonso García-Parrado ${ }^{1}$ and José M Senovilla, Causal structures and causal boundaries", 2005 Class. Quantum Grav. 22 R1; https://arxiv.org/abs/gr-qc/0501069

10. Carter B (1971) “Causal structure in Space-time” Gen. Rel. Grav. 1349-391

11. Ibrahim Dincer and Yunus A. Cengel, "Energy, Entropy and Exergy Concepts and Their Roles in Thermal Engineering", Entropy 2001, 3(3), 116-149;

12. Øystein Elgarøy ,* and Øyvind Grøn, "Gravitational entropy and inflation", Entropy 2013, 15(9), 3620-3639

13. Edward Kolb, And Michael Turner," The Early Universe", CRC Press, Boca Racon, Florida, USA, 2018

14. Ng, Y.Jack Entropy 2008, 10(4), 441-461; DOI: $10.3390 / \mathrm{e} 10040441$

15. Lloyd , Seth "Computational capacity of the universe", Phys. Rev. Lett. 88, 237901 (2002), https://arxiv.org/pdf/quant-ph/0110141.pdf

16. Seth Lloyd, "Ultimate physical limits to computation”, https://cds.cern.ch/record/396654/files/9908043.pdf

17. Zeh, H. D (2010). The Physical Basis of The Direction of Time. ISBN 978-3-540-42081-1

18. Ioannis Haranas1 and Ioannis Gkigkitzis," The Mass of Graviton and Its Relation to the Number of Information according to the Holographic Principle", Hindawi Publishing Corporation International Scholarly Research Notices Volume 2014, Article ID 718251, 8 pages http://dx.doi.org/10.1155/2014/718251 
19. Beckwith, A.W. (2018) Initial Conditions for Defining an Arrow of Time at the Start of Inflation? Journal of High Energy Physics, Gravitation and Cosmology, 4, 787-795. https://doi.org/10.4236/jhepgc.2018.44044

20. E. Kolb, and S. Turner "The Early Universe", Westview Press, Chicago, USA, 1994 .

21. Andreas Albrecht, "Cosmic inflation and the arrow of time", https://arxiv.org/abs/astro-ph/0210527

22. Katherine Freeze, Mathew Brown, and William Kinney, "The Phantom Bounce, A new proposal for an oscillating Cosmology", pp 149-156, of "The Arrows of Time, A debate in cosmology", Fundamental theories in physics, Volume 172, with Laura Mercini-Houghton, and Rudy Vaas, editors, Springer Verlag, Heidelberg, Federal Republic of Germany, 2012

23. Maxim Kohopov, "Fundamentals of Cosmic Particle physics", Cambridge international science publishing, Cambridge, UK, 2012

24. S.W. Hawking,"Arrow of time in Cosmology", Phys. Rev. D 32, 2489 - Published 15 November 1985

25. Mario Castagnino , Olimpia Lombardi, Luis Lara, "The arrow of time in cosmology", http://philsci-archive.pitt.edu/800/1/The Arrow of Time in Cosmology.pdf

26. Serguei Krasnikov, "Back in time and faster than light travel in General Relativity", Fundamental theories of Physics, 193, Springer Nature, Cham, Switzerland, 2018

27. Albecht Lindner and Dieter Strauch, "A complete course on theoretical physics", Undergraduate Lecture Notes in Physics, Springer Nature, Cham, Switzerland, 2018

28. Roger Penrose (2006). "Before the Big Bang: An Outrageous New Perspective and its Implications for Particle Physics"(PDF). Proceedings of the EPAC 2006, Edinburgh, Scotland: 2759-2762.

29. Richard Tolman, Relativity, Thermodynamics, and Cosmology. Oxford: Clarendon Press. 1934. LCCN 34032023. Reissued (1987) New York: Dover ISBN 0-486-65383-8.

30. Helge Kragh*" Cyclic models of the relativistic universe: the early history", https://arxiv.org/ftp/arxiv/papers/1308/1308.0932.pdf

31. Lohn D. Barrow (Author), Erank J. Tipler (Author), Lohn A. Wheeler (Foreword)," The Anthropic Cosmological Principle (Oxford Paperbacks) Revised ed. Edition, Oxford University Press, Oxford, UK, 1996

32. Stephen Hawking, "Black holes, and Baby Universes, and other essays" Bantam books, New York, New York, USA, 1994

33. Andrew Walcott Beckwith, "Using "Enhanced Quantization" to Bound the Cosmological Constant, (for a Bound-on Graviton Mass), by Comparing Two Action Integrals (One Being from General Relativity) at the Start of Inflation", pp 21-36, of "Fundamental Physics and Physics Education Research", editors of Burra G. Sidharth • Jesús Carnicer Murillo • Marisa Michelini • Carmen Perea, Published by Springer Nature Switzerland AG 2021, in Gewerbestrasse 11, 6330 Cham, Switzerland

34. Penrose, Roger (1965), "Gravitational collapse and space-time singularities", Phys. Rev. Lett., 14 (3)

35. Natário, J. (2006). "Relativity and Singularities - A Short Introduction for Mathematicians". Resenhas.6: 309-335. arXiv:math.DG/0603190

36. Poplawski, N. (2011) Cosmological Constant from QCD Vacuum and Torsion. Annals of Physics (Berlin), $523,291$. https://doi.org/10.1002/andp.201000162

37. Andrei Linde and Vitaly Vanchurin, "How many universes in the multiverse", https://arxiv.org/pdf/0910.1589.pdf

38. P. Halmos, Ergodic theory, Publ. Math. Soc. Japan,No. 3, Math. Soc. Japan,Tokyo, 1956

39. 39.V. I. Arnold and A. Avez, Ergodic Problems of Classical Mechanics, (1968) W. A. Benjamin, Inc.

40. .Omri Sarig," Lecture Notes on Ergodic Theory", March 8, 2020, http://www.weizmann.ac.il/math/sarigo/sites/math.sarigo/files/uploads/ergodicnotes.pdf

41. LECTURES BY MARYAM MIRZAKHANI NOTES BY TONY FENG, "INTRODUCTION TO ERGODIC THEORY", https://www.mit.edu/ fengt/ergodic theory.pdf

42. Miao Li (Author), Xiao-Dong Li (Contributor), Shuang Wang (Contributor), Yi Wang (Contributor), "Dark Energy", Peking University Press, World Scientific, Singapore, Republic of Singapore, 2015

43. M. Novello and R. P. Neves, "The mass of the graviton and the cosmological constant," Classical and Quantum Gravity, vol. 20, no. 6, pp. L67-L73, 2003.

44. Padmanabhan, Thanu, "An Invitation to Astrophysics", World Press Scientific, World Scientific Series in Astronomy and Astrophysics: Volume 8, Singapore, Republic of Singapore, 2006

45. Nathan Rosen," Quantum Mechanics of a Miniuniverse", International Journal of Theoretical Physics, Vol. 32, No. 8, 1993

46. Ioannis Haranas1 and Ioannis Gkigkitzis," The Mass of Graviton and Its Relation to the Number of Information according to the Holographic Principle", Hindawi Publishing Corporation International Scholarly Research Notices Volume 2014, Article ID 718251, 8 pages http://dx.doi.org/10.1155/2014/718251

47. Andrei Linde,"The inflationary Multiverse", pp 127-149 of "Universe or Multiverse", edited by Bernard Carr, Cambridge University Press, New York City, New York, USA, 2007 